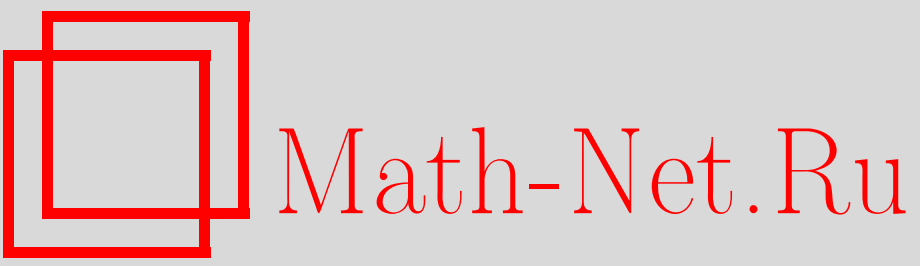

М. Н. Богачева, И. В. Павлов, О хааровских расширениях безарбитражных финансовых рынков до безарбитражных и полных, УМН, 2002, том 57, выпуск $3,143-144$

DOI: https://doi.org/10.4213/rm515

Использование Общероссийского математического портала Math-Net.Ru подразумевает, что вы прочитали и согласны с пользовательским соглашением

http://www. mathnet.ru/rus/agreement

Параметры загрузки:

IP: 34.229 .108 .108

26 апреля 2023 г., 04:25:48 


\title{
О ХААРОВСКИХ РАСШИРЕНИЯХ БЕЗАРБИТРАЖКНХ ФИНАНСОВЫХ РЫНКОВ ДО БЕЗАРБИТРАЖНЫХ И ПОЛНЫХ
}

\author{
М. Н. БОГАЧЁВА, И. В. ПАВЛОВ
}

Настоящая работа связана с вопросом преобразования неполных рынков в полные. Впервые такого рода задача была рассмотрена в [1], где переход от неполных рынков к полньм осуществлялся заменой исходной мартингальной меры неэквивалентной ей матрингальной мерой. Однако с помощью полученной таким образом единственной мартингальной меры невозможно вычислять цены финансовых контрактов, справедливые для изначально рассматриваемого финансового рьнка. Этот недостаток впервые был преодолен в работе [2] (см. также [3]). В этой работе пополнение финансового рынка проводилось посредством добавления к рисковым активам исходного рынка дополнительных активов, функционально зависимых с изначальными.

В докладах [4], [5] была заложена основа принципиально другого метода перехода от неполных рынков к полным - метода интерполяции. Данная статья посвящена изложению результатов, полученных в этом направлении и являющихся окончательными для модели с конечным вероятностным пространством и конечным горизонтом.

Пусть $(\Omega, \mathscr{F})$ - измеримое пространство с конечной $\sigma$-алгеброй $\mathscr{F} ; \mathscr{P}$ - множество вероятностных мер $\mathrm{P}$ на $(\Omega, \mathscr{F})$, нагружающих все атомы $\sigma$-алгебры $\mathscr{F} ; \mathbf{F}=\left(\mathscr{F}_{k}\right)_{k=0}^{N}$ - филштрация, удовлетворяющая условиям $N<\infty, \mathscr{F}_{0}=\{\Omega, \varnothing\}$ и $\mathscr{F}_{N}=\mathscr{F} ; Z=\left(Z_{k}, \mathscr{F}_{k}\right)_{k=0}^{N}-\mathbf{F}$-адаптированный случайньй процесс; $\mathscr{P}(Z, \mathbf{F})$ - множество таких мер $\mathrm{P} \in \mathscr{P}$, для которых процесс $\left(Z_{k}, \mathscr{F}_{k}, \mathrm{P}\right)_{k=0}^{N}$ является мартингалом; $|\mathscr{P}(Z, \mathbf{F})|$ - число элементов множества $\mathscr{P}(Z, \mathbf{F})$. Пусть

$$
\begin{gathered}
A=B_{1}+B_{2}+\cdots+B_{m}, \\
a:=\left.Z_{k}\right|_{A}, \quad b_{i}:=\left.Z_{k+1}\right|_{B_{i}},
\end{gathered}
$$

где $A$ - атом в $\mathscr{F}_{k}(0 \leqslant k<N), B_{i}$ - атомы в $\mathscr{F}_{k+1}(i=1,2, \ldots, m ; 1 \leqslant m<\infty)$.

Также рассмотрим на $(\Omega, \mathscr{F})$ так называемую хааровскую фильтрацию $\mathbf{H}=\left(\mathscr{H}_{n}\right)_{n=0}^{L}\left(\mathscr{H}_{0}=\right.$ $\left.\{\Omega, \varnothing\}, \mathscr{H}_{L}=\mathscr{F}\right)$, характеризуемую следующим свойством: при любом $n \sigma$-алгебра $\mathscr{H}_{n}$ порождена разбиением $\Omega$ ровно на $n+1$ атом. Хааровскую фильтрацию Н будем называть интерполирующей хааровской фильтрацией для фильтрации $\mathbf{F}$, если существует последовательность натуральных чисел $0=n_{0}<n_{1}<\cdots<n_{N}:=L$, для которой $\mathscr{H}_{n_{k}}=\mathscr{F}_{k} \forall k(0 \leqslant k<N)$.

Предположим, что $\mathrm{P} \in \mathscr{P}(Z, \mathbf{F})$ и $\mathbf{H}$ - интерполирующая хааровская фильтрация для $\mathbf{F}$. Рассмотрим случайную величину $Y_{n}=\mathrm{E}^{P}\left[Z_{N} \mid \mathscr{H}_{n}\right]$ и процесс $Y=\left(Y_{n}, \mathscr{H}_{n}\right)_{n=0}^{L}$, интерполирующий процесс $Z$ в том смысле, что $Y_{n_{k}}=Z_{k}$. Будем говорить, что мартингальная мера $\mathrm{P} \in \mathscr{P}(Z, \mathbf{F})$ удовлетворяет свойству хааровской единственности (CXE), если для филштрации $\mathbf{F}$ можно построить такую хааровскую интерполяцию $\mathbf{H}$, что для соответствующей мартингальной интерполяции $Y=\left(Y_{n}, \mathscr{H}_{n}\right)_{n=0}^{L}$ имеет место соотношение $|\mathscr{P}(Y, \mathbf{H})|=1$.

Теорема 1. 1) Если какая-нибудь мера $\mathrm{P} \in \mathscr{P}(Z, \mathbf{F})$ удовлетворяет $C X E$, то для всех $k(0 \leqslant k<N)$ и любого атома $A \in \mathscr{F}_{k}$ при $m>1$ выполняется неравенство

$$
\min _{1 \leqslant i \leqslant m} b_{i}<a<\max _{1 \leqslant i \leqslant m} b_{i}
$$

а при $m=1-$ равенство

$$
a=b_{1} .
$$

2) Если $\forall k(0 \leqslant k<N)$ и для любого атома $A \in \mathscr{F}_{k}$ при $m>1$ выполняется неравенство (2), а при $m=1$ - равенство (3), то любая мера $\mathrm{P} \in \mathscr{P}(Z, \mathbf{F})$ удовлетворяет CXE.

СлЕДСТвИЕ 1. Если существует $\mathrm{P} \in \mathscr{P}(Z, \mathbf{F})$, удовлетворяющая $C X E$, то любая $\mathrm{P} \in \mathscr{P}(Z, \mathbf{F})$ удовлетворяет $C X E$.

Работа выполнена при поддержке Российского фонда фундаментальных исследований (грант № 02-01-00909). 
Введем теперь условие, более сильное, чем условие хааровской единственности. Будем говорить, что мера $\mathrm{P} \in \mathscr{P}(Z, \mathbf{F})$ удовлетворяет свойств у универсальной хааровской единственности (СУХЕ), если для любой хааровской интерполяции $\mathbf{H}$ фильтрации $\mathbf{F}$ и соответствующей мартингалшной хааровской интерполяции $Y=\left(Y_{n}, \mathscr{H}_{n}\right)_{n=0}^{L}$ мартингала $Z=\left(Z_{k}, \mathscr{F}_{k}\right)_{k=0}^{N}$ имеет место равенство $|\mathscr{P}(Y, \mathbf{H})|=1$.

Пусть $\left(b_{1}, b_{2}, \ldots, b m\right)$ - конечный набор действительных чисел, снабженных, соответственно, весами $\left(p_{1}, p_{2}, \ldots, p_{m}\right)$, где $0<p_{i}<1, i=1,2, \ldots, m$. Будем говорить, что этот набор чисел удовлетворяет условию несовпадения барищентров (УНБ), если для любых двух непересекающихся подмножеств индексов $I=\left\{i_{1}, i_{2}, \ldots, i_{\alpha}\right\} \subset\{1,2, \ldots, m\}$ и $J=\left\{j_{1}, j_{2}, \ldots, j_{\beta}\right\} \subset$ $\{1,2, \ldots, m\}$ выполнятся неравенство:

$$
\frac{b_{i_{1}} p_{i_{1}}+b_{i_{2}} p_{i_{2}}+\cdots+b_{i_{\alpha}} p_{i_{\alpha}}}{p_{i_{1}}+p_{i_{2}}+\cdots+p_{i_{\alpha}}} \neq \frac{b_{j_{1}} p_{j_{1}}+b_{j_{2}} p_{j_{2}}+\cdots+b_{j_{\beta}} p_{j_{\beta}}}{p_{j_{1}}+p_{j_{2}}+\cdots+p_{j_{\beta}}} .
$$

Предположим, что мы находимся в рамках обозначений (1), и пусть $p_{i}=P\left(B_{i}\right)$.

Tеорема 2. Мера $\mathrm{P} \in \mathscr{P}(Z, \mathbf{F})$ удовлетворяет СУХЕ тогда и только тогда, когда $\forall k(0 \leqslant k<N)$ u для любого атома $A \in \mathscr{F}_{k}$ набор чисел $b_{1}, b_{2}, \ldots, b_{m}$, снабженных весами $p_{1}, p_{2}, \ldots, p_{m}$, удовлетворяет УНБ.

Teорема 3. Пусть $\mathscr{P}(Z, \mathbf{F}) \neq \varnothing$. В множестве $\mathscr{P}(Z, \mathbf{F})$ есть меры, удовлетворяющие СУХЕ, тогда и только тогда, когда $\forall k(0 \leqslant k<N)$ и для любого атома $A$ из $\mathscr{F}_{k}$ числа $b_{1}, b_{2}, \ldots, b_{m}$ различны и ни одно из них не совпадает с числом $a$.

Если предположить, что в обозначениях (1) $m$ всегда меньше или равно 3 , то можно получить следующее усиление теоремы 3.

Tеорема 4. Пусть $\mathscr{P}(Z, \mathbf{F}) \neq \varnothing u \forall k(0 \leqslant k<N)$ nрu переходе от $k \kappa k+1$ любой атом $A$ из $\mathscr{F}_{k}$ дробится не более чем на три атома (т.е. $m \leqslant 3$ ). Тогда при выполнении условий теоремы 3 любая мартингальная мера $\mathrm{P} \in \mathscr{P}(Z, \mathbf{F})$ удовлетворяет CYXE.

Teорема 5. Пусть $\mathscr{P}(Z, \mathbf{F}) \neq \varnothing$ и существует $k(0 \leqslant k<N)$ u атом $A$ из $\mathscr{F}_{k}$, который при переходе от момента $k$ к моменту $k+1$ дробится более чем на три атома (m.е. $m \geqslant 4)$. Тогда существует $\mathrm{P} \in \mathscr{P}(Z, \mathbf{F})$, не удовлетворяющая CУХЕ.

Покажем далее, как изложенная выше идеология может применяться к исследованию финансовых рьнков. На фильтрованном пространстве $\left(\Omega, \mathscr{F}, \mathscr{F}_{k}\right)_{k=0}^{N}$ рассмотрим $(B, S)$-рынок, где положительные процессы $B=\left(B_{k}, \mathscr{F}_{k}\right)_{k=0}^{N}$ и $S=\left(S_{k}, \mathscr{F}_{k}\right)_{k=0}^{N}$ отражают эволюцию, соответственно, цены банковского счета и цены акции. Обозначим $Z_{k}=S_{k} / B_{k}$. Предположим, что данный $(B, S)$-рьнок безарбитражен, т.е. $\mathscr{P}(Z, \mathbf{F}) \neq \varnothing$. Однако в болшшинстве случаев такие рынки неполны, т.е. $|\mathscr{P}(Z, \mathbf{F})|=\infty$. Хорошо известно, что все расчеты (вычисление цен опционов, расчет хеджирующих стратегий и т. д.) в рамках неполных рынков значительно сложнее, чем в полных. Изложенная вьше методика указьвает один из возможных путей сведения неполного рынка к полному. Именно, процесс $Y=\left(Y_{n}, \mathscr{H}_{n}\right)_{n=0}^{L}$ интерполирует дисконтированный процесс $Z$ на "промежуточные времена". Так как мы считаем, что в промежуточные времена проценты в банке не начисляются, то естественно положить $\widetilde{B}_{n}=B_{k}$ при $n_{k} \leqslant n<n_{k+1}$ $(0 \leqslant k<N)$ и $\widetilde{B}_{L}=B_{N}$. Таким образом, интерполяция цен акций на промежуточные времена определяется формулой: $\widetilde{S}_{n}=\widetilde{B}_{n} Y_{n}$ при $0 \leqslant n \leqslant L$.

\section{СПИСОК ЛИТЕРАТУРЫ}

[1] M.S. Taqqu, W. Willinger // Adv. Appl. Probab. 1987. V. 19. P. 1-25. [2] А. В. Мельников, К. М. Феоктистов // Обозрение прикл. промышл. матем. 2001. Т. 8. № 1. С. 28-40. [3] K. М. Феоктистов // УМН. 1998. Т. 53. № 6. С. 165-166. [4] M. N. Bogacheva, I. V. Pavlov // International Conference "Stochastic Analysis and Related Topics": Abstracts of communications. St. Petersburg, 2001. P. 13-14. [5] М.Н. Богачёва, И. В. Павлов // Обозрение прикл. промышл. матем. 2001. Т. 8. № 1. С. 107-108. 